Quelques métaphores, au miroir des analyses spatiales : réseaux de villes et réseaux de pouvoir

Jean-Pierre Gaudin et Denise Pumain

\title{
CpenEdition
}

Journals

Édition électronique

URL : http://journals.openedition.org/ress/714

DOI : $10.4000 /$ ress.714

ISSN : 1663-4446

Éditeur

Librairie Droz

Édition imprimée

Date de publication : 1 février 2000

Pagination : 105-119

ISBN : 2-600-00409-2

ISSN : 0048-8046

Référence électronique

Jean-Pierre Gaudin et Denise Pumain, «Quelques métaphores, au miroir des analyses spatiales :

réseaux de villes et réseaux de pouvoir », Revue européenne des sciences sociales [En ligne],

XXXVIII-117 | 2000, mis en ligne le 17 décembre 2009, consulté le 20 avril 2019. URL : http://

journals.openedition.org/ress/714; DOI : 10.4000/ress.714 


\section{QUELQUES MÉTAPHORES, AU MIROIR DES ANALYSES SPATIALES : réseaux de villes et réseaux de pouvoir}

Comment et pourquoi la notion de réseau est-elle devenue une métaphore des relations interurbaines, des échanges de personnes, de biens et d'informations entre les villes, et d'une composition volontaire et rationnelle des fonctions urbaines? On se souvient en effet de la célébration intense des figures du «réseau de villes » ou du «réseau urbain » à partir des années 60-70. Quel miroir nous tendent-elles aujourd'hui pour réfléchir à la manière dont se pensent les rapports entre espace et politique?

Le réseau est étymologiquement d'abord un filet et a pu devenir, en tant que tel, le support d'un premier usage analogique possible. Mais par glissement rapide, le contenant s'est projeté sur le contenu et le réseau initial des fils a pu faire penser, par exemple, à des maillages de canalisations ou de voies diverses, puis à des graphes de relations de toute nature. La métaphore du réseau est donc devenue essentiellement relationnelle.

De manière générale, la métaphore suggère, séduit mais laisse beaucoup de significations en suspens. Reconnaissons-lui cette force de l'ambiguïté mais tentons, en même temps, de la décrypter. Quelle est la nature des relations interurbaines visées, en l'occurrence, par l'image du réseau? Ce qui est de l'ordre de la circulation ne s'inscrit pas dans n'importe quelle règle d'échange. Et les rapports hiérarchiques peuvent être aussi bien masqués, dans certains cas, que revendiqués, dans d'autres situations. D'autant que, à travers des variations sémantiques légères (réseaux de villes, réseaux urbains, armatures urbaines) et des transferts de références et d'images, cet usage métaphorique des réseaux articule subtilement le monde de la connaissance scientifique et celui de l'action, le descriptif et le prescriptif.

L'histoire des usages de la métaphore réticulaire dans l'analyse spatiale et les politiques d'aménagement amène ainsi à reconsidérer tout à la fois le classique regard rationnel et surplombant de l'aménageur étatique qui triomphe dans les années 60-70, puis l'interpellation plus récente des villes en tant que sujets de leur propre développement lorsque l'esprit de marché et de concurrence s'est développé, mais également les chassés-croisés les plus actuels entre ce qui reste d'un volontarisme planificateur d'ensemble et l'engouement croissant pour des «partenariats» négociés ponctuellement.

Circulations interurbaines, représentations de relations, métaphores de réseaux, il nous faut donc interroger ces variations autour d'un type de savoir sur l'espace mêlé à une famille de politiques publiques, en partant d'une période qui, en France par exemple, commence au milieu des années 60. Il se dégagera de cet examen que la métaphore du «réseau de villes» a pour l'essentiel abrité deux 
figures qui se voulaient distinctes mais qui sont en réalité étroitement jumelées par leurs enjeux: celle de la relation et la circulation, d'une part; celle de la domination et la hiérarchie, d'autre part. Ce savoir métaphorique et ses usages politiques sont cependant aujourd'hui en nette évolution: on commentera donc deux perspectives d'un possible destin ultérieur de ces métaphores urbaines et urbanistiques: le réseau interurbain de négociation et le réseau bio-technologique comme métaphore des relations urbaines.

\section{1. - LE «RÉSEAU», MÉTAPHORE DES RELATIONS ET DES CIRCULATIONS INTERURBAINES}

\section{Configurations}

La notion de « réseau de villes », entendue à la fois comme grille de lecture des semis de villes mais aussi comme objectif de planification, apparaît en France comme une sorte de co-production à la fin des années 60 entre universitaires (géographes et économistes) et aménageurs de la Délégation à l'aménagement du territoire.

Les géographes avaient, pour leur part, développé préalablement le terme de «réseaux urbains» dans une perspective plus purement analytique. Cela traduisait, après-guerre, un premier repositionnement significatif de la géographie française dans la façon de penser les relations villes-campagnes (Chabot, 1954). Plutôt, en effet, que de s'appuyer principalement sur les découpages physiques ou paysagers et sur les activités agricoles, comme il était courant dans la géographie régionale française, le raisonnement partira des activités urbaines et, bientôt, de l'idée de «réseaux urbains».

A partir des années 60 , le contexte d'ensemble est alors marqué par une croisade nouvelle en faveur d'une «géographie appliquée» ou «active» (Labasse, 1955; George et al., 1964; Phlipponeau, 1960), c'est-à-dire d'une géographie focalisée sur les développements de l'urbanisation et susceptible d'apporter ses expertises aux urbanistes et aux aménageurs. Elle prend appui sur des travaux d'économistes et de géographes allemands (Christaller, 1933) ou américains (Berry, 1971) mais aussi sur ceux de certains économistes français (Piatier, 1956; Ponsard, 1955; Boudeville, 1961).

Pour les «géo-aménageurs » (cf. les travaux de Rochefort, 1960, et de Hautreux, 1965), raisonner dans la perspective métaphorique du «réseau urbain» comportait plusieurs implications importantes et répondait à différents objectifs :

- l'accent pouvait être mis sur des flux de personnes et d'information, en termes de critères de flux de relations, et sur des pôles d'échange (donc, des liens et des nœuds de réseaux);

- on envisageait ainsi les relations directes des villes avec leur territoire (hinterland, «zones de desserte») mais surtout celles des villes entre elles;

- on situait, ce faisant, très directement un savoir géo-économique par rapport à des objectifs d'action (développement de la politique d'aménagement du territoire, de rééquilibrage régional et de rationalisation des niveaux d'équipements urbains après 1963). Dans le même temps, en adoptant une posture 
hypothético-déductive (c'est la desserte des usagers, liée elle-même au poids des populations, qui exprime et structure des aires d'influence et conduit à définir des niveaux différenciés de services collectifs), une génération de jeunes géographes, souvent proches du marxisme, se différenciait de ses aînés et de la tradition plutôt inductive de la géographie française.

\section{Enjeux}

Cette figure contemporaine du réseau «urbain » ou « de villes » se rattache toutefois à une tradition métaphorique ancienne qui applique l'image du réseau à des relations qui se traduisent par des circulations de biens, de personnes ou d'informations. Pareille figuration s'organise volontiers soit selon une logique ramifiée en arbre, soit selon une composition de lignes entrecroisées et formant boucle. Ainsi en va-t-il de la physiologie de la circulation du sang, tout d'abord, à partir du XVIII ${ }^{\mathrm{e}}$ siècle. Puis des voies des communication: télégraphe (1849), téléphone (1879), chemin de fer, seulement ensuite, semble-t-il (1870), transport fluvial (1924) et enfin aérien (début du XX $\mathrm{XX}^{\mathrm{e}}$ siècle). Dans cette perspective, sont progressivement englobés tant des réseaux techniques que les systèmes de services qui y sont associés. Et la liaison des deux éléments se resserre encore avec l'image encore plus puissante des réseaux informatiques contemporains.

La problématique des «réseaux» de villes, pour sa part, se situe à l'intersection des deux perspectives que ménage la métaphore des réseaux, l'une suggérant une certaine hiérarchie, une composition ramifiée en arbre ou bien une image de fleuve accueillant ses affluents (ce que suggèrent également les visualisations de réseaux urbains sous forme de graphes pyramidaux), tandis que l'autre n'évoque simplement que des connections et des nœuds d'échange. Or la notion de réseau de villes va jouer en fait pleinement de l'ambiguïté de ces deux connotations possibles.

L'analogon, l'effet de ressemblance, peut en effet être double. Il est d'une part connoté par une logique d'esprit gravitaire (Lösch, Christaller). Les villes sont alors des planètes d'importance diverse qui s'attirent et se contrôlent mutuellement en particulier selon des effets de taille de population, (concentrée ou bien desservie dans une «zone d'influence») ayant recours à des services collectifs concentrés. L'effet hiérarchique est alors à la fois présent mais en quelque sorte naturalisé par l'analogie gravitaire et, donc, socialement euphémisé. Cette métaphore astronomique ne date pas d'hier: le géographe du roi G. Sanson en 1681 appliquait la classification que Ptolémée réservait aux étoiles aux villes pour en hiérarchiser la magnitude et l'éclat (cité par Lamarre, 1998). Ce n'est cependant que bien plus tard qu'est apparue l'analogie entre réseau de villes et galaxie, par exemple chez Reclus (1905 ) et Levasseur (1889-92).

L'ambiguïté produite peut être d'autant plus grande en matière de réseau urbain que, d'autre part, la métaphore du réseau se rattache, plus largement, à une perspective simplement relationnelle, celle de l'échange et de la circulation, laquelle reste indéterminée sur la question de la domination. On notera à ce propos, car on ne peut oublier que les villes sont aussi des lieux politiques, que les analyses classiques de l'échange politique (Hobbes, avec la philosophie politique contractualiste, Pizzorno, avec l'échange dans le cadre de l'Etat social, et Marin avec l'échange politique généralisé) laissent, elles aussi, ouvertes toutes les possi- 
bilités, depuis l'échange inégal qui traduit les rapports de domination jusqu'aux formes coopératives de collaboration qui seraient marquées par la confiance et le caractère «horizontal» des relations de pouvoir.

Par conséquent, l'approche en termes de «réseaux de villes » laisse voir plusieurs usages analogiques potentiels, qui cultivent largement l'ambiguïté de par leurs inspirations multiples et la richesse de leurs connotations. Cela ne peut qu'inciter à mieux cerner les enjeux de pouvoirs qui ont pu se situer derrière l'utilisation un peu irénique de l'image du réseau, dans le contexte particulier des politiques d'aménagement et d'urbanisme des années de croissance : pouvoirs concurrentiels des universitaires, pouvoirs des administrations de mission face aux grands ministères, pouvoirs conflictuels des maires urbains chacun soucieux d'autonomie. L'effet-miroir de la métaphore peut souvent devenir un effet-masque.

\section{2. - LES IMPLICITES DE LA MÉTAPHORE RÉTICULAIRE : FRONTIÈRES, DOMINATIONS, HIÉRARCHIES DES VILLES}

Les réseaux, comme on le sait, sont aussi des rets en vieux français, des filets, et ils peuvent donc suggérer l'image de l'emprisonnement, comme dans les rets de l'amour célébrés au XVII ${ }^{\mathrm{e}}$ siècle... La métaphore du réseau rejoint ici le schème de la «couverture » du territoire, qui sous-tendait dès le XVII ${ }^{\mathrm{e}}$ siècle les représentations de l'aménagement des places-fortes militaires, avant celles de la desserte par les commerces et les services (Pumain, Robic, 1996). En tout cas, le terme n'est jamais à lui seul la garantie d'une égalité dans les relations ou la figure d'un club d'égaux, c'est-à-dire un ensemble de pôles réunis par des liens horizontaux.

\section{Configurations}

La genèse du terme éclaire-t-elle le sens donné à la métaphore? L'expression de «réseau de villes», évoquée par P. Vidal de la Blache (1898) et formalisée par G. Chabot (1948) à partir des travaux de W. Christaller, avait été conçue par les géographes pour dépasser l'approche monographique d'une ville dans sa région et construire un niveau d'observation supérieur, en inventant un nouvel objet, une nouvelle échelle d'analyse du fait urbain. Le terme se cherche dans la littérature scientifique, Jean Tricart dans son cours de 1954 hésite encore entre réseau de villes et semis urbain, et c'est clairement Michel Rochefort qui l'impose dans son article des «Annales de Géographie» en 1957: «Le réseau urbain défini, dans les limites régionales de l'influence de la grande ville capitale, par l'existence et la localisation d'un certain nombre de types de centres-relais, eux-mêmes hiérarchisés, qui constituent les mailles du réseau » (p. 125).

La structure hiérarchique d'un ensemble de centres urbains différenciés par le niveau (rareté, diversité et nombre) de leurs fonctions est ainsi clairement associée à la définition des réseaux urbains. Elle se lit aussi dans les différences d'intensité et les dissymétries des flux échangés entre les villes. Et c'est bien dans ce sens de la hiérarchisation et du contrôle des flux que la géographie urbaine va être amenée à mobiliser l'expression de réseau urbain, au moment où elle se rapproche des préoccupations d'aménagement du territoire des années 60 (M. Rochefort surtout, qui fait alliance avec le polytechnicien J. Hautreux). 
Les réseaux de villes deviennent «réseaux urbains », donc, dans le vocabulaire de l'action. Mais plus spécifiquement apparaissent aussi les termes «armature urbaine», «hiérarchie urbaine», avec, à la clé, quelques notions secondaires explicites: «zones de commandement» ou d'influence... On est là assez loin des réseaux d'allure horizontale, qui auraient pu trouver des références historiques dans certaines ligues urbaines du passé comme celle de la Hanse. Il s'agit ici d'une problématique de la catégorisation des différences et des niveaux, pour une plus claire hiérarchisation des équipements collectifs et une concentration des efforts dans les dépenses publiques. Sans atteindre le caractère systématique des classifications de centres urbains en trois niveaux hiérarchisés, telle qu'elle est définie par exemple pour l'affectation des équipements publics par les Länder allemands, la sélection de huit «métropoles d'équilibre » en 1964 va dans le sens de la bataille menée par l'Etat central pour le développement local et la re-localisation des activités. Il n'est pas non plus anodin que l'expression d' "armature urbaine», apparue dans un texte du géographe G. Chabot en 1948, ait été préférée à celle de réseau urbain dans les discussions du Commissariat au Plan entre géographes et ingénieurs, selon G. Mercadal (1965), afin d'éviter la confusion possible avec les réseaux techniques urbains. La conception, normative et figée, de l'armature urbaine hiérarchisée qui corsète le territoire, prend alors le pas sur les connotations circulatoires attachées à la métaphore du réseau.

\section{Enjeux}

C'est là une stratégie du contrôle pyramidal de l'espace et plus précisément des villes secondaires par les métropoles. Les armatures, définies autour du poids des populations, des niveaux de services, des flux de communications et quelques aires d'attraction, seront en effet mises en relation avec des normes de planification et des «grilles» d'équipements, non sans un certain mépris, dans les années qui précèdent la loi de décentralisation de 1982, des cultures politiques spécifiques et des pouvoirs politiques locaux.

Dans cette problématique tutélaire des armatures urbaines, popularisée par un aménagement du territoire triomphant, que devient alors le pouvoir urbain? Il est fortement contraint par des tutelles multiples, tant juridiques que financières et techniques. Il doit composer avec le pouvoir central qui détient encore l'essentiel des moyens financiers et économiques de la production urbaine de l'époque. Mais il tente également de ruser avec la logique dominante de hiérarchisation et de coopérations obligées. Néanmoins, l'esprit du temps est à rebours.

C'est là en effet que se dévoile l'intérêt d'opérer des déplacements, des métaphores, qui, en dépit ou bien à cause de l'avertissement d'Aristote, qui se méfiait déjà méthodologiquement du procédé, autorisent plus encore que l'analogie (c'est-à-dire la ressemblance) des formes de transposition, des jeux d'images, des allusions suggestives - ce dont rend compte la progressive «navigation » sémantique du terme réseau à travers l'histoire du français.

En effet, la circulation et les échanges, ce qui faisait simplement image suggestive, ou ressemblance, dans la première approche de la notion de réseau urbain (ou de villes), laisse place ici à une véritable stratégie de la métaphore, c'est-à-dire l'imposition d'une conception hiérarchique et emboîtée des relations. Elle admet 
comme une fatalité les forces gravitationnelles, elle souligne la nécessité des coopérations et complémentarités de voisinage, dans un système pyramidal.

Trois intentions majeures semblent guider cette démarche des réseaux urbains durant les Trente glorieuses:

- en dosant habilement, tout d'abord, les critères qui se rapportent aux réseaux matériels (communications physiques, flux de populations et de clientèles) avec ceux qui concernent des réseaux immatériels (information, contrôle administratif), on promeut la nouvelle modernité des communications et on banalise une mobilité plus intense;

- de plus, en privilégiant les «métropoles d'équilibre», on commence de mettre l'accent sur des régions, à une échelle plus vaste et fonctionnelle que celle des départements napoléoniens;

- enfin, en valorisant des indicateurs relatifs aux activités de services, on emphatise le passage d'une économie du primaire et du secondaire à une économie tertiaire (Boudeville, 1961). A cet égard, l'acceptation du terme de «réseau urbain» en quelque sorte «naturalise» des grilles d'équipement hiérarchisées et permet de normaliser certains arbitrages.

Au total, un regard surplombant est jeté sur les pouvoirs locaux qui s'obstinent dans des concurrences de clochers et qui sont, sinon sommés, du moins fortement incités à s'associer. Ce régime de coopérations obligées peut avoir des conséquences importantes sur la régulation des rapports ami/ennemi, qui tient un rôle fondamental dans le jeu politique.

Les nouveaux réseaux de villes, que l'on promeut à la DATAR, à l'INSEE et dans les travaux de quelques géographes, dans les années 1990 (DATAR, 1991) vont-ils supprimer les effets de frontières précédemment entretenus entre villes voisines rivales? Les effets de gaspillage liés à la duplication des équipements et aux compétitions interurbaines sont alors vivement critiqués au nom d'un discours de raison qui, en réalité, ne supprime pas les concurrences et les frontières mais cherche à les organiser à une nouvelle échelle. En ce sens, les réseaux de villes alors promus ne sont pas «supérieurs » aux frontières et aux démarcations, ils en dessinent une nouvelle géographie, qui intègre des méga-régions et déjà l'échelle européenne. La règle du jeu est ainsi changée et confirmée tout à la fois.

Pourtant, le vocabulaire de la géographie ne sort pas indemne de ses échanges avec celui des politiques d'aménagement du territoire. L'ambiguïté des notions d'armature et de réseau, l'usage banalisé de ce dernier terme qui tend à s'opposer à la notion de hiérarchie, commencent dès les années 1980 à contaminer le vocabulaire scientifique, et, dans la notion de réseau urbain hiérarchisé, l'image d'une hiérarchie de pouvoir tend à se substituer à celle des niveaux de complexité des activités tertiaires que reconnaissait la théorie des lieux centraux. Ainsi trouve-ton curieusement ce double reniement assumé dans le dictionnaires de Roger Brunet (1992): «les géographes parlent volontiers de 'réseau urbain' pour qualifier l'ensemble des villes d'un pays. Il arrive que ce ne soit pas un vrai réseau [sic], mais un simple arbre hiérarchique, lorsque les villes ne communiquent qu'avec la capitale, et n'échangent guère directement entre elles». Ainsi désormais pour les géographes une hiérarchie ne serait plus une forme particulière de réseau, et l'em- 
ploi de réseau urbain ou réseau de villes renverrait à la notion a-hiérarchique d'association volontaire de villes telle que préconisée par la DATAR... Ces glissements sémantiques vont obliger à changer le vocabulaire scientifique pour en développer l'intuition initiale. On peut se demander si c'est le statut de «science molle» qui, en autorisant le maintien prolongé d'une définition semi-métaphorique de concepts partagés avec le politique, a pu conduire à une mainmise de celui-ci sur la signification du langage commun? Autre effet de frontière et de domination...

\section{3. - LES RÉSEAUX DE VILLES \\ ET LA NÉGOCIATION ENTRE LE MÊME ET L'AUTRE}

La conjonction étroite de l'aménagement du territoire années 70 et de la problématique géographique des réseaux de villes a légitimé l'affirmation d'un regard surplombant du pouvoir central sur l'ensemble du pays et, en parallèle mimétiquement, de chacune des métropoles secondaires sur leurs espaces régionaux.

\section{Configurations}

Ce regard de l'Etat, en quelque sorte «ethnocentrique», puisqu'il projette ses propres catégories sur l'autre, a eu une force certaine, bien qu'il ait rencontré progressivement des obstacles croissants à mesure que les mesures décentralisatrices entraient en actes. Certes, les villes proches et de rang égal étaient supposées s'allier en «régions de villes», plutôt que de se concurrencer sur des équipements de haut niveau, s'associer en somme comme des entreprises aux créneaux complémentaires. Mais les villes étaient avant tout censées tenir chacune leurs zones d'influence, tout comme les capitales nationales commandent leurs territoires. La logique dominante de représentation de l'espace et la conception du pouvoir (économique comme politique) était donc d'abord d'ordre pyramidal et unifiant. Dans cette approche des réseaux urbains, via la pyramide organisée de l'armature urbaine, la capitale tendait en quelque sorte un miroir aux métropoles régionales, où l'image de l'autre (les villes de rang secondaire) n'était plus que le modèle réduit de l'image de soi.

Cependant, ce volontarisme rationalisateur de l'aménagement du territoire, qui cherchait à typifier les niveaux d'équipement et à catégoriser les aides publiques afin de lutter contre le saupoudrage des crédits publics, a trouvé d'emblée des limites qui sont allées ensuite croissant: rivalités politiques locales durables, actualisées par les compétitions nouvelles sur les relocalisations des activités industrielles et tertiaires; logiques administratives de «guichet» qui se reconstituent; concurrences renouvelées en termes d'équipements, focalisés toujours plus sur les loisirs, la culture et le tourisme; contournements de la planification des équipements par des subventions spécifiques. Tout cela avant même le développement des effets vigoureusement concurrentiels de la décentralisation et du néo-libéralisme.

Cela signait-il, dès lors, la fin progressive d'un certain volontarisme d'aménagement, d'incitations planifiées à une rationalisation accrue de la hiérarchie 
urbaine et, indirectement, d'une «géographie utile» tournée vers l'aide à l'action publique?

Le planisme spatial, qui intégrait réseaux urbains et régions de villes, s'est peut-être un peu dilué dans les pays où les gouvernements avaient été initialement les plus interventionnistes mais il s'est aussi pour partie reporté sur des schémas d'aménagement plus sectoriels (transports, infrastructures scolaires ou sanitaires, etc.) et, plus encore, il a été transposé à un échelon où le volontarisme conserve d'autant plus de marges de manœuvre qu'il reste mieux à distance des logiques politiques locales : celui de l'Europe. Le document du Schéma de Développement de l'Espace Communautaire adopté à Potsdam en mai 1999 (ESDP, 1999) insiste sur un «développement spatial polycentrique » pour lequel les politiques préconisent de ne plus seulement relier les périphéries au centre mais d'assurer l'intégration internationale des régions périphériques et de leurs métropoles en s'appuyant sur des réseaux de villes («city networks» est l'expression employée dans la traduction anglaise). C'est volontairement euphémiser à nouveau la hiérarchie.

\section{Enjeux}

Mais s'agit-il là seulement d'une sorte de repli tactique, d'un changement d'échelle du volontarisme appliqué aux réseaux urbains, ou encore d'une simple nuance apportée aux ambitions initiales? On peut en réalité plutôt voir dans ce repositionnement la conséquence d'une nouvelle conception émergente de l'action publique en général et de l'aménagement en particulier. On a en effet progressivement pris conscience que les réseaux de villes ne sont pas nécessairement faits que de circulations de flux de biens ou de personnes, ou encore que de maillages techniques. On peut les voir aussi comme des ensembles de relations sociales, des systèmes relationnels, des «réseaux » sociaux et politiques.

Il est de fait au sein des sciences sociales une autre approche possible des réseaux que celle qu'on a privilégiée longtemps dans l'analyse des systèmes urbains. Elle s'est développée depuis le début du siècle, dans une perspective qui se dégage rapidement des échanges matériels et des infrastructures, même si elle s'enracine par ses présupposés dans la même métaphore circulatoire (réseau sanguin). Mais elle est sociologique, plutôt d'esprit interactionniste et a priori n'implique pas de relation non hiérarchique (Mauss, 1924; Radcliffe-Brown et l'anthropologie culturelle anglo-saxonne, Moreno et la psychologie expérimentale américaine des années 30).

Les «social networks » ont d'abord été envisagés comme des réseaux de sociabilité, en somme d'appropriation du monde social et d'expression des affinités ou amitiés. L'analyse de réseau s'est ensuite développée en termes de sociologie des organisations et de sociologie politique, dans l'étude des phénomènes croisés de coopération et de concurrence (par exemple Crozier et Friedberg, 1981; Mabileau, 1992).

Les réseaux de villes, perçus comme étant des réseaux politiques et sociaux, constituent une perspective d'analyse ou d'action qu'on a tendance aujourd'hui à prendre plus largement en compte, lorsqu'on l'on veut se départir de l'attitude quasiment magique des précédentes politiques d'aménagement, celles qui postulaient des rationalités surplombantes et des solidarités obligées. C'est en particulier ce que montre l'approche italienne maintenant célèbre (et un peu mythifiée à 
son tour) des districts industriels et les stratégies de développement local qui y sont associées (Bagnasco, 1977). Les effets imputables à la proximité spatiale s'avèrent être autant d'effets dus à des réseaux culturels, professionnels, familiaux, confessionnels, etc. De ce fait, la démarche de modélisation «aménagiste » devient autre, ne peut se contenter d'images spatiales rationnelles mais doit compter avec des dynamiques sociales et politiques inscrites dans une temporalité et des processus culturels parfois de longue durée. La problématique du réseau intègre dès lors une pluralité de références et une négociation devenue nécessaire entre une multiplicité d'acteurs locaux et centraux, politiques et sociaux, institutionnels et associatifs.

Devant cette évolution récente, on peut alors avancer une question. A travers un pareil changement de sensibilité et de problématisation des approches, n'est-ce pas une nouvelle normativité qui s'invente? Appliquée aux villes comme à d'autres objets susceptibles d'aménagements (voire à d'autres politiques publiques), l'approche des «réseaux» sociaux-politiques cherche en effet à tenir compte et même à mobiliser largement les dynamiques potentielles des acteurs sociaux, à les impliquer par un mélange d'incitations et d'offre de procédures négociées, au lieu de leur prescrire ou leur imposer une rationalité donnée (Gaudin, 1999). De ce fait, la problématique des réseaux sociaux s'inscrit dans une perspective plus vaste qui, aujourd'hui, vise à privilégier une régulation de caractère multi-centré, se déployant à l'échelle des régions de villes ou bien, plus finement, à celle des «pays», des «communautés de villes »...

Le réseau (de villes) devient par conséquent porteur d'une nouvelle métaphore, celle du partenariat négocié et des arrangements technico-politiques.

On ne peut cependant conclure que cette perspective d'évolution soit unique. Simplement, elle peut se déployer parallèlement aux démarches volontaristes des schémas d'aménagement classiques qui perdurent dans des domaines «lourds » de planification des dépenses publiques (infrastructures). Basée sur une conception processuelle et réticulée de la production des normes sociales, elle laisse place à la logique du contrat et du projet à côté, par exemple, de celle des organisations stables (structures intercommunales ou régionales), des planifications «compréhensives » ou globales. Si ce n'est pas là nécessairement une pleine reconnaissance de l'Autre, ce n'est plus, du moins, l'enfermement dans le Même, dans la reproduction des catégories imposées par une rationalité surplombante. Faire réseau, réseauter entre forces politiques et sociales qui négocient des objectifs partageables et une production normative conjointe devient cohérent avec un système décisionnel multi-centré subsidiaire, comme dans les pays fédéraux ou décentralisés.

\section{4. - LA TENTATION PROPHÉTIQUE ET LES RÉSEAUX BIO-TECHNOLOGIQUES}

Malmené dans ses concepts par les captures langagières du politique, le discours savant a développé de nouvelles stratégies métaphoriques. Depuis une ou deux décennies, des images circulent dont aucune ne s'est vraiment imposée comme le nouvel avatar métaphorique du réseau de villes. Bien que d'inspirations différentes, elles ont en commun de véhiculer des connotations assez éloignées 
des processus sociaux, et de les «naturaliser» ou de les instrumentaliser. Aux fonctions précédentes, elles ajoutent peut-être ce que l'on pourrait qualifier superficiellement et de façon anecdotique un «effet Sokal», en s'appropriant le vocabulaire des sciences de la complexité. On fait ici l'hypothèse que ces images conduisent vers de nouvelles métaphores des réseaux de villes qui tireraient leur force évocatrice des sciences cognitives, associant l'adaptabilité biologique et la technicité informatique.

Cette évolution a été préparée de longue date par la substitution de l'expression de «système de villes » à celle de réseau urbain. On peut remarquer au passage une différence intéressante, mais encore non expliquée, entre les usages français et anglo-saxons de ces deux termes. Dans un contexte d'une grande densité de références croisées entre les lexiques urbains français et anglo-saxons au cours des années 1950-60 (Pinchemel, 1983), il faut souligner l'opposition entre la diffusion, en France, d'expressions construites sur le mot de «réseau » et son inexistence dans la littérature anglo-saxonne au profit du terme de system. Dans le champ de définition des relations interurbaines, les expressions de urban network et de networks of towns n'ont pas «pris » et c'est la terminologie de central place theory, ou central place hierarchy, pattern, model, spacing... qui a prévalu, à partir des années cinquante et de la reconnaissance de la théorie de Christaller par la new geography, suivie de celle des systèmes de villes (Pumain, Robic, 1999, 75).

Peut-être l'usage américain a-t-il influencé peu à peu la pratique française. Mais c'est aussi contre l'image fixe, les connotations statiques attachées au réseau, que la notion de système de villes a été adoptée (Pumain, 1992). Elle accroche des images de transformations, de changements interdépendants, de coévolutions. La métaphore spatiale se teinte alors de temporalité, elle appelle les formalisations de la dynamique et de la complexité. Elle s'adapte aussi aux changements de paradigme scientifique ambiants, entre les années 1960 et 1980-90, pour délaisser les déterminations rigoureuses (mais déjà non linéaires, autorisant les effets contre-intuitifs) des systèmes à la Forrester (1964) et adopter les aléas, les turbulences et les bifurcations des systèmes auto-organisés (Pumain et al., 1989; Sanders, 1992). Certains y ajoutent volontiers le chaos. D'autres s'irritent de ces analogies trop physiciennes qui oublient les finalités de l'acteur, ses intentions et les effets de pouvoir, pour ne retenir que l'émergence fatale de structures d'ensemble contraignantes mais non concertées sur fond de concurrences individuelles atomisées (Lepetit, Pumain, 1993). En décrivant la structure comme le résultat non intentionnel et souvent non perçu de multiples actions et interactions interindividuelles, la métaphore du système auto-organisé place en effet l'objet réseau de villes hors de portée de l'intervention directe du politique régulateur ou planificateur, elle relativise l'action de chacun dans une multitude complexe de régulations locales dont la résultante globale est pratiquement imprévisible. La métaphore du système de villes en tant que système auto-organisé aurait-elle alors une fonction «déresponsabilisante»? Est-ce pour cette raison qu'elle ne s'est encore guère imposée dans les milieux de l'aménagement du territoire, hormis pour désigner des ensembles supra-régionaux de villes dépendantes d'une grande métropole, à la DATAR vers le début des années 1990?

Par rapport à la notion de réseau, on peut aussi souligner que celle de système de villes tend à s'abstraire du territoire qui en constitue le support ou qu'il s'agit de desservir. Ce sont en effet surtout les interrelations urbaines, qui, progressive- 
ment, déterminent le devenir des villes, au fur et à mesure que les fonctions de production industrielle et de services ont pris le pas sur celle de la production agricole. Ce déracinement urbain est évoqué d'une autre manière, en opérant un retour vers une géographie physique, par l'image de l'archipel qu'emploie P. Veltz (1996) pour qualifier l'économie contemporaine. Il donne ainsi à voir la matérialité des concentrations urbaines, comme des concrétions émergées de leur territoire, noyaux durs d'une économie dégagée du substrat de la terre agricole ou d'un arrière-pays à desservir et contrôler et vouée dans sa quasi-totalité au seul service des citadins. Les villes apparaissent comme des îlots d'activité connectés dans une surface qui serait indifférenciée, voire liquidée. Cette image inverse curieusement le rapport entre les villes et l'eau - ne disait-on pas que les villes, par ailleurs si souvent situées sur des rivières, irriguaient leur territoire? - et édulcore singulièrement l'analogie qui liait les organisations urbaines et leurs dépendances hiérarchiques à la forme arborescente et collectrice des réseaux hydrographiques (Chorley, 1964). Elle substitue aussi à l'unidirectionnalité des écoulements la liberté des circulations océanes, et aux régularités christallériennes l'ordre aléatoire et lâche (quoique, en fait, tout aussi fractal que celui des villes) des émergences telluriques.

La prochaine métaphore des réseaux urbains se cherche, et sans prétendre à la divination, ni même vouloir tenter une prédiction auto-réalisatrice, nous pensons qu'elle pourrait bien s'inspirer de la cognitique. Une telle image aurait des chances de succès car elle fusionnerait deux sources analogiques jusqu'ici fécondes, celle de la biologie et celle des nouvelles technologies.

On sait que la métaphore biologique a sa tradition urbaine, d'abord explicitement organiciste (Mackinder, 1902) puis peu à peu dégagée de ses connotations naturalistes, quand le systémisme évolue vers les théories de l'auto-organisation. Les jalons sont multiples, depuis la «théorie des générations de villes » de Mackinder, qui invoque une «sélection naturelle» d'inspiration darwinienne, jusqu'à l'image du système nerveux chez Geddes, en passant par celle de la métropole régionale, «organe directeur», dont la nodalité représente l'émergence d'un niveau de complexité d'organisation, chez Vidal de la Blache (Robic, 1989 a et b). La référence à la biologie courait dans mainte monographie régionale sous les images partielles, et aujourd'hui vieillies, des villes évoquées comme le système nerveux (ou artériel) qui impulse l'activité et la nouveauté dans les territoires. L'image a récemment été réactivée par celle de la «synapse», suggérée en 1990 par R. Brunet pour évoquer les lieux de l'échange et les «villes de contact». C'est pour l'opposer à la hiérarchie que R. Brunet emploie à propos des villes l'image d'un «système neuronal» dans un ouvrage de 1994 sur l'aménagement du territoire...

La métaphore organiciste est l'une des trois figures récurrentes employées à propos des systèmes de villes, avec celles du réseau d'étapes et de la couverture du territoire (Pumain, Robic, 1996). On a vu que l'une des fonctions principales de ces métaphores est d'édulcorer les rapports de pouvoir et la très forte différenciation hiérarchique qui caractérisent les relations entre les villes. Si ces métaphores se parent actuellement d'une scientificité empruntée à l'extérieur des sciences sociales, c'est peut-être aussi en raison de la résistance de cet objet à se laisser approcher et gouverner. Le vocabulaire tend à en faire une entité complexe, vivante, qui évoluerait selon ses lois propres et cachées. 
L'idée de la complexité s'est encore insérée dans l'image du réseau de villes par une autre voie, celle de l'analogie technique. Le courant favorable au progrès technique, dont la mode récurrente est sans doute à l'origine du succès de la métaphore du réseau à l'époque des chemins de fer, s'est emparé à nouveau de la notion en la modernisant. P. Claval (1982) avait ainsi suggéré l'image du commutateur téléphonique pour définir une ville qui «maximise les interactions sociales» par le branchement des individus les uns avec les autres, selon une combinatoire démultipliée de la mise en relation.

C'est vers une fusion new age entre le biologique et le technique que s'orientent désormais les chercheurs d'image. La cognitique, qui rapproche le fonctionnement du cerveau et celui des ordinateurs, la physiologie nerveuse et les puces électroniques, via les processus d'apprentissage et l'intelligence artificielle, fournit le terrain idéal. Dans le monde branché sur le virtuel, il n'y a pas loin de la synapse à l'interface, et la souplesse adaptative du vivant jointe à l'efficacité des réseaux informatiques se prêtent à des allusions qui se veulent riches de sens.

C'est ainsi que tente de s'imposer une image de la ville tout à la fois mémoire, gisement de savoir-faire et créatrice, c'est la «city of knowledge » dont l'avènement est prophétisé par exemple par R. Knight. Là encore, le glissement métaphorique, des réseaux de transport aux réseaux d'information, ne manque pas d'oblitérer les relations de pouvoirs, et ce risque n'a pas échappé à C. Raffestin: «on peut se demander si à l'ancien despotisme oriental des sociétés hydrauliques ne correspondrait pas, toutes choses égales par ailleurs, un despotisme occidental des sociétés informationnelles » (1988, p. 272). C'est ainsi encore que sont tentées des analogies assez directes entre la représentation informatique des réseaux neuronaux et celle des réseaux de villes (Buscema, Diappi, 1999). La métaphore se glisse derrière l'apparence opérationnelle de son analogue informatique, en dissimulant dans la boîte noire des couches neuronales, sous forme de processus parallèles et de connexions intermittentes, périodiquement réactivées, les interdépendances floues, apparemment indéterminées, entre des acteurs sociaux que l'on voudrait rendus aux libertés de la pensée, dégagés des contraintes spatiales et des effets de pouvoir, imprégnés de connaissance, mais mettant en œuvre quelle volonté, quelle intelligence supérieure?

A côté des réseaux de neurones viendra peut-être une image du réseau de villes comme hypertexte, instrument d'une circulation librement choisie entre des nœuds de savoir, et de création par l'interconnexion et la navigation (nous retrouvons là l'image de l'archipel) entre des centres de ressources, d'informations et de connaissances. Les «nomades urbains» ne surfent-ils pas déjà d'une ville à l'autre, entre les miroirs identitaires ou dépaysants qu'elles proposent, à travers les systèmes de signes, les images qu'elles se donnent dans la maîtrise concertée des opérations de marketing urbain? L'organisation de cette production d'image autour d'un projet de ville entre des partenaires locaux rejoint l'hypothèse optimiste évoquée ci-dessus à propos du Même et de l'Autre, en laissant augurer d'une meilleure prise en compte de la diversité, de l'autonomie et de l'inventivité des acteurs locaux, voire des simples citoyens citadins.

La fonction de ces nouvelles métaphores, le réseau neuronal ou l'hypertexte, serait donc double: d'une part elles serviraient à naturaliser les idées d'autoapprentissage, d'adaptation, de flexibilité, désormais requises de la part des acteurs urbains, censés réagir avec une conscience plus précise des interdépen- 
dances multiples dans lesquelles se situe leur action; d'autre part, et de manière plus ambiguë, s'installe une représentation de la ville comme sujet, image organiciste récurrente qui sous l'avatar du réseau apprenant en édulcore les possibles conflits d'intérêt.

\section{CONCLUSION}

Le succès de la métaphore du réseau urbain vient peut-être de sa particulière richesse sémantique, qui a autorisé son utilisation aussi bien pour justifier la soumission au regard surplombant de l'aménageur national que pour inciter l'initiative locale en coopération. C'est sans doute aussi que l'objet ainsi construit n'est pas familier, il ne relève guère d'une perception immédiate. On ne commence à l'apercevoir que sur les cartes à petite échelle, il atteint le grand public avec les cartes routières et certaines images de satellite. Ce n'est pas un hasard si les premières analogies des géographes ont rapproché au XVII ${ }^{\mathrm{e}}$ siècle les réseaux de villes, invisibles, des étoiles, bien visibles (Lamarre, 1998). Il n'y a donc pas eu d'appréhension directe, non savante, des réseaux de villes (contrairement à la catégorie du paysage par exemple). Dans le langage savant même, la notion de réseau urbain reste bien la contribution spécifique des géographes, des politologues et des aménageurs à la réflexion qui pour d'autres se limite à «la» ville.

La petite histoire des « récupérations-transformations» des métaphores scientifiques par des acteurs politiques peut être lue comme une nouvelle illustration de la difficulté du passage de la connaissance à l'action, et de sa légitimation. Elle souligne aussi, en creux, la nécessité d'une production métaphorique périodiquement renouvelée entre les deux mondes, instrument de communication nécessaire entre des réseaux sociaux qui ne partagent pas toujours les mêmes références.

En effet, à côté du classique débat relatif aux rapports entre métaphore et rationalité scientifique, il en est un autre que nourrit plus particulièrement notre cas de figure. Longtemps et dans des champs très divers, d'Aristote à Claude Bernard, la pensée scientifique s'est méfiée de la métaphore, source d'imprécision et plus suggestive que démonstrative. A présent, et notamment avec Jean-Claude Passeron, des réévaluations s'opèrent: la démarche métaphorique peut être aussi considérée comme une source irremplaçable d'inventivité dans les sciences historicosociologiques et comme un moyen puissant de transfert de raisonnements entre disciplines. Mais reste une autre question, un peu différente, celle de la place de la métaphore dans les rapports entre connaissance et action. Là encore, on peut avancer qu'elle autorise des décloisonnements. Elle est en effet une des figures possibles du transfert ou de la «traduction» de questions et de notions entre mondes de légitimité différents; elle favorise aussi des «hybridations » entre normes ou systèmes de référence (B. Latour). En somme, on ne peut plus s'en tenir aujourd'hui au cloisonnement des rationalités entre le «savant» et le «politique», cher à Max Weber dans les années vingt. Pour autant, les métaphores, dans ce cas, s'avèrent être trop souvent des passerelles fragiles et dangereuses. Les images circulent d'autant plus facilement qu'elles sont floues, les mots se diluent et, par réaction, requièrent in fine de nouvelles constructions terminologiques. Déconnectés, en attendant, de leur contexte et de leurs cadres de référence méthodologiques ou théoriques, les termes métaphoriques deviennent des sortes de notions 
orphelines. Et surtout, comme on l'a aperçu précédement, la rigueur de la construction intellectuelle s'efface trop derrière le travail de séduction du financeur de recherches ou d'études.

L'évolution des connotations et des mots et expressions ainsi associés à la métaphore du réseau urbain paraît guidée par trois fonctions parfois contradictoires: la première met à jour les contenus des interprétations en fonction de la transformation des faits sociaux, des attributs caractéristiques des villes; la seconde intègre un certain progrès des connaissances scientifiques en caractérisant le réseau comme le résultat de processus, d'évolutions interdépendantes, audelà de l'image fixe de l'armature et des infrastructures, en faisant évoluer la notion du réseau vers celle des systèmes de villes; la troisième est peut-être la fonction plus incantatoire, mais sans doute auto-réalisatrice, de la reconnaissance du nécessaire partenariat politique.

C'est d'ailleurs seulement par le contenu social et politique instillé dans ces métaphores qu'on peut imaginer que l'objet réseau de villes, ainsi représenté de façon partagée, pourrait être sinon maîtrisé, du moins gouverné avec une conscience plus élaborée de ses possibilités. Encore faut-il que les métaphores du réseau, y compris dans leurs avatars les plus récents, n'occultent pas complètement les réalités des hiérarchies et du pouvoir, en masquant des stratégies d'évitement et d'ignorance des forces d'initiatives et de la diversité locales.

Centre comparatif d'étude des politiques publiques (CEPEL)

CNRS - Université de Montpellier

et

Département de géographie

Université de Paris 1 - Panthéon-Sorbonne

\section{RÉFÉRENCES}

Bagnasco A., Le tre Italie, Il Mulino, 1977.

Berry B., Géographie des marchés et du commerce de détail, Paris, A. Colin, 1971.

Boudeville J.R., Les espaces économiques, Paris, PUF, 1961.

Brunet R., La France, un territoire à ménager, Paris, Editions 1, 1994.

Brunet R., Ferras R., Théry H., Les mots de la géographie, Montpellier, RECLUS, 1992.

Buscema M., Diappi L., La struttura complesse delle citta : un approcio cognitivo basato su reti neurali, in «Cybergeo», 100, 1999, 28 p.

Chabot G., Géographie des villes, Paris, A. Colin, 1948.

Chabot G., L'armature urbaine en géographie régionale, in Urbanisme et architecture, 1954.

Chorley R.J., Geography and Analogue Theory, in «Annals of the Association of American Geographers», 54, 1964, pp. 127-137.

Christaller W., Die Zentralen Orte in Süddeutschland, Iena, Fischer, 1933.

Claval P., La logique des villes, Paris, LITEC, 1982.

Crozier M., Friedberg E., L'acteur et le système, Paris, Le Seuil, 1977.

DATAR, En Europe, des villes en réseaux., Paris, La Documentation française, 1991, 109 p.

ESDP, European Spatial Development Perspective, Towards Balanced and Sustainable Development of the Territory of the EU, Potsdam, May 1999, 82 p. 
Forrester J., Urban Dynamics, MIT Press, 1964.

Gaudin J.P., Gouverner par contrat. L'action publique en question, Presses de sciences politiques, 1999.

George P., Guglielmo R., Kayser B., Lacoste Y., La géographie active, Paris, PUF, 1964.

Hautreux J., Rochefort M., Physionomie générale de l’armature urbaine française, in «Annales de géographie », 74, 1965.

Labasse J., Les capitaux et la région, Paris, A. Colin, 1955.

Lamarre C., La ville des géographes de l'époque moderne, XVI ${ }^{e}$-XVIII ${ }^{e}$ siècle, in "Genèses », 32 , 1998, pp. 4-27.

Lepetit B., Pumain D. (coordinateurs), Temporalités urbaines, Paris, Anthropos, Coll. Villes, 1993, $318 \mathrm{p}$.

Levasseur E., La population française. Histoire de la population française avant 1789 et démographie de la France, 3 vol., 1889-1892.

Mabileau A., Le système politique local, Paris, Montchrétien, 1992.

Marin B., Generalized Political Exchange, Westview Press, 1991.

Mauss M., Essai sur le don, in «L'année sociologique», 1924.

Mercadal G., Les études d' armature urbaine régionale, in «Consommation», 3, 1965, pp. 3-42.

Phlipponeau M., Géographie et action, Paris, A. Colin, 1960.

Piatier J., L'attraction commerciale des villes, in « Revue juridique et économique du Sud-Ouest», 1956.

Pinchemel P. et G., Geography and the City. A Contribution to the History of Geography in France, in Patten J. (ed), The Expanding City. Mélanges offerts au Professeur Jean Gottman, London, Academic Books, 1983, pp. 295-318.

Pizzorno A., Political Exchange and the Collective Identity in Industrial Conflict, in Crouch A.P., The Resurgence of Class Conflict in Western Europe, MacMillan, 1978.

Ponsard C. 1955, Economie et espace, SEDES.

Pumain D., Sanders L., Saint-Julien T., Villes et auto-organisation. Paris, Economica, 1989.

Pumain D., Les systèmes de villes, in Bailly A., Ferras R., Pumain D. (éd.), Encyclopédie de géographie, Paris, Economica, 1992, chap. 34, pp. 645-664.

Pumain D., Les modèles d'auto-organisation et le changement urbain, in «Cahiers de Géographie de Québec », 42, 1998, 117, pp. 349-366.

Pumain D., Robic M.-C., Théoriser la ville, in Derycke P.H., Huriot J.M., Pumain D., Penser la Ville, Théories et modèles, Paris, Anthropos,, 1996, chap. 4, pp. 107-161.

Pumain D., Robic M.-C., Réseaux urbains, des mots aux choses, in «Urbanisme», 304, 1999, pp. 72-75.

Raffestin C., Repères pour une théorie de la territorialité humaine, in G. Dupuy (dir.), Réseaux territoriaux, Caen, Paradigmes, 1988, pp. 263-279.

Reclus E., The Evolution of Cities, in «The Contemporary Review », 67, 2, 1895, pp. 246-264.

Reclus E., L'Homme et la Terre, Librairie Universelle, 1905.

Reynaud J., Villes, in Encyclopédie Nouvelle, t. VIII, Gosselin, 1841.

Robic M.-C., Métropole/métropôle. Les géographe et les métropoles d'équilibre, in «Strates», 4, 1989 (a).

Robic M.-C., Perspectives temporelles sur l'émergence de quelques concepts de la géographie urbaine française, in «Sistemi Urbani », 3,1989, pp. 225-239 (b).

Rochefort M., Méthodes d'analyse des réseaux urbains. Intérêt de l'analyse du secteur tertiaire de la population active, in «Annales de Géographie», 1957.

Rochefort M. 1960, L'organisation urbaine de l'Alsace, Université de Strasbourg (thèse de doctorat ès lettres et sciences humaines), 1960.

Sanders L., Systèmes de villes et synergétique, Paris, Anthropos, 1992.

Veltz P., L'économie d'archipel, Paris, Editions de l'Aube, 1996.

Vidal de la Blache P., La géographie politique d'après les écrits de M. Frédéric Ratzel, in «Annales de Géographie», 1898. 